\title{
Lebensqualität statt Warenberge
}

\author{
Wer die unterschiedlichsten Produkte erwerben oder verschiedene Dienstlei- \\ stungen in Anspruch nehmen will, hat heutzutage bei der Beschaffung wenig \\ Probleme. Selbst der Preis ist bei vielen Produktgruppen angesichts von \\ Marktsättigung und Massenfertigung meist kein Hemmnis mehr. Mit immer \\ marktschreierischer Werbung und ausgefalleneren Arten der Finanzierung ver- \\ sucht man den Konsum anzuschieben.
}

$\mathrm{K}$ Von Georg Abel onsum heißt heute: Wir leisten uns Dinge, die wir uns nicht leisten können. Wir überbewerten ihre wirkliche Bedeutung für uns und wir unterbewerten die sozialen, gesundheitlichen und ökologischen Folgen. Unser Glücksgefühl ist abhängig vom Kauf eines toten, aber zeitgemäßen Gegenstandes. Dabei verschließen wir die Augen davor, daß der Preis viel zu hoch ist. Damit ist nicht nur die steigende private oder öffentliche Verschuldung gemeint, sondern auch die psychische Verelendung und zunehmende Verarmung der Gesellschaft sowie der Raubbau an der Natur oder die Zerstörung der Ressourcen kommender Generationen.

Den typischen Konsumenten gibt es heute nicht mehr. Die Konsumforschung wird heute mit unterschiedlichsten Konsumententypen konfrontiert. Dabei verschieben sich für einen Teil der Verbraucher die Konsumwerte: War früher der Fernseher oder später das Auto Statussymbol für die breite Masse, stellt ein Teil der Konsumenten heute diese „Immer-mehr-, Immerschneller-Mentalität" in Frage und achtet auf andere Qualitäten von Produkten: beispielsweise die ökologisch korrekte Herstellung der Produkte oder den fairen Handel. Die Zahl dieser „Moral-Plus-Konsumenten“ wächst.

Doch eine Romantisierung der Konsumenten ist nicht angesagt. Trotz enormen Umweltbewußtseins klafft zwischen Bewußtsein und tatsächlichem Verhalten eine große Lücke. Das „Volk der Getrenntsammler" ist - insgesamt betrachtet - von einem ökologischen Konsum noch weit entfernt. Ansätze wie der Verzicht aufs Privatauto und Angebote wie Car-Sharing sind zwar vorhanden und erfreuen sich zunehmender Nachfrage, doch insgesamt steigt die Zahl der zugelassenen Autos weiter. Und wer sich das gute Gewissen über das Kompostieren holt, fliegt dann gern auch mal etwas weiter. Kaum ein Reiseprospekt oder Reiseveranstalter, der nicht das Schlagwort „Sanfter Tourismus“ verwendet. Und wer kennt nicht auch in der Ökoszene die Macher, die schnell mal in den Flieger springen. Das mag zynisch klingen, aber die Zahl der CarSharing-Mitglieder, der Marktanteil von fairgehandeltem Kaffee oder der Absatz ökologischer Lebensmittel ist vorsichtig formuliert noch deutlich steigerbar. Andere Länder sind hier schon ein Stück weiter. In Österreich oder Dänemark werden ökologisch erzeugte Lebensmittel nicht nur im Fachhandel vertrieben. Dort treten die Bioprodukte direkt im Supermarkt gegen konventionell erzeugte Produkte an, der Marktanteil liegt in diesen Ländern deutlich über dem Anteil in Deutschland.

\section{Zielgruppe Unternehmen}

Gesättigte und oft auch rückläufige Märkte bieten hier aber Chancen. Wo die Produkte immer ähnlicher werden und sich Marktanteile nur im Verdrängungswettbewerb erzielen lassen, verschiebt sich die Marktmacht zugunsten der Verbraucher. Je gesättigter der Markt desto geringer braucht die Zahl entschlossener Käufer zu sein, um Unternehmen zu Veränderungen $\mathrm{zu}$ motivieren. Will Shell seine Plattform billig im Atlantik entsorgen, zwingt das Anfahren anderer Tankstellen das Unternehmen zum Einlenken. Der BSE-Skandal sorgt für den Schwund des Rindfleischkonsums und für Umsatzsteigerungen bei den ökologischen Anbauverbänden. Unternehmen werden künftig immer mehr ökologische und gesellschaftspolitische Verantwortung übernehmen müssen. Meinungsumfragen zeigen, daß die Bereitschaft, Kaufentscheidungen mit umweltpolitischen und sozialen Wertentscheidungen zu koppeln, gestiegen ist.

Für eine ökologische Verbraucherorganisation ergibt sich daraus die Aufgabe, den Verbrau-

\section{DIE ERDE GIBT KEINEN KREDIT MEHR}

540 Mrd DM kostet die Dekontaminierung der durch die Reaktorkatastrophe in Tschernobyl verseuchten Gebiete. 150 Mrd DM sind für die Befestigung gefährdeter US-Küsten erforderlich, wenn der Meeresspiegel infolge der globalen Erwärmung um einen Meter steigt. 190 Mrd DM sind für die Abwasserbeseitigung in Deutschland bis zum Jahre 2000 notwendig. 39 Mrd DM kostet die Erhaltung von 2.000 Tierarten mit jeweils 500 Exemplaren die nächisten 20 Jahre.

Die wachsende Menschheit lebt mit immer schneller schwindenden Naturressourcen. Der Übergang vom Jahrhundert der industriellen Evolution in das Jahrhundert der Umwelt erfordert eine Orientierung an neuen Zielen und Märkten und andere Wirtschaftsweisen, bei denen der Umweltschutz von der Reparaturabteilung in die Konstruktionsabteilung einer Gesellschaft verlegt wird.

Die Fachzeitschrift "Blick durch Wirtschaft und Umwelt" zeigt Entwicklungen auf und liefert Fakten und Hintergründe für ein künftiges Umwelt-Management und strategisches Handeln.

Mit dem Bestellcoupon können Sie Ihr persönliches Probeheft anfordern.

\section{BESTELLCOUPON}

\section{"Blick durch}

Wirtschaft und Umwelt"

Media Point Verlag

Postfach 9102 40, 90260 Nürnberg

Bitte schicken Sie mir kostenlos die aktuelle Ausgabe zum Kennenlernen:

\section{Name}


chern konkrete Verhaltensvorschläge und praktikable Handlungsalternativen vorzustellen. Ökologisch-soziale Verbraucherpolitik muß aber auch auf die Grenzen des Konsums hinweisen, muß die Auswirkungen unseres hemmungslosen Konsums für die Menschen der zweiten und dritten Welt, für die kommenden Generationen und für die Armen der ersten Welt darstellen.

Der kritische Dialog mit Unternehmen und Handel wird für die ökologisch-soziale Verbraucherpolitik an Bedeutung gewinnen. Die Möglichkeiten dafür haben sich durch den Generationenwechsel in den Unternehmen erhöht, und damit bietet sich die Chance einer Auseinandersetzung jenseits des klassischen Freund-Feind-Denkens. Konfrontative Auseinandersetzungen mit Unternehmen bis zum Boykott werden aber nicht an Bedeutung verlieren.

\section{- Sustainable Consumption}

Viel ist in den letzten Jahren über Sustainable Development geredet worden. Trotz der unterschiedlichen Vorstellungen dazu ist eines klar: Die bloße Übertragung der Produktions- und Konsumweise der Industrie auf die Entwicklungsländer funktioniert nicht. Sie ist aus ökologischen und sozialen Gründen zum Scheitern verurteilt. Die Konsequenz daraus muß ein Umsteuern vor allem in den entwickelteren Ländern sein. „Wohlstand für alle“ lautete der Titel eines 1958 erschienenen Buches von Ludwig Erhard. Zwar erreicht heute der materielle Wohlstand ein damals unvorstellbares Niveau, aber Rekordarbeitslosigkeit und zunehmende Armut und Verschuldung sind heute die andere Seite dieser Medaille.

Sustainable Consumption bedeutet in diesem Zusammenhang ein neues Bild des Konsums. Gefragt ist nicht ein Konsum, der sich an kurzfristigen und individuellen Motiven ausrichtet, sondern der Blick in die Zukunft und dabei die Orientierung an den Bedürfnissen auch der kommenden Generation. Es geht um langfristige, kollektive oder globale Interessen und Bedürfnisse.

Damit dieses Ziel erreicht werden kann, brauchen wir eine Konsumwende: Lebensqualität, Zufriedenheit und Wohlstand müssen nach diesem neuen Leitbild nicht an immer mehr Güterverbrauch gekoppelt sein. Dabei kommt sowohl den Verbrauchern als auch den Unternehmen die Aufgabe zu, gesellschaftliche Verantwortung wahrzunehmen. Der Umbau unserer Industrie- gesellschaft zu einer von allen Akteuren akzeptierten Gesellschaft mit sozialen und ökologischen Schwerpunkten bedeutet einen generellen Wandel und ist nicht durch Einzelmaßnahmen zu erreichen.

In Hinblick auf diese Weiterentwicklung der Industriegesellschaft kommt drei Bereichen besondere Bedeutung zu:

- Das Wegwerfprinzip ist out, Minimierung des Ressourcenverbrauchs ist in. Ökologisch und ökonomisch sinnvoll sind langlebige, reparaturfreundliche, wiederverwertbare Produkte. Die Entlastung der Umwelt wird hier für die Verbraucher mit dem Produktnutzen verbunden, ohne daß auf Lebensqualität verzichtet werden muß. Zusätzlich muß darüber nachgedacht werden, wie das Prinzip ,teilen statt besitzen“ gefördert werden kann. Warum sollen zukünftig nur Autos gemeinsam genutzt werden und nicht auch Waschmaschinen etc.?

- Die Ernährung muß sich ändern. Umweltschonende Landwirtschaft, artgerechte Tierhaltung und regionale Produkte sind hier die Stichworte einer gesundheitlich unbedenklichen Ernährung.

- Die Energiewende muß her. Eine andere Energiepolitik wird drastische Auswirkungen auf Konsum-, Wohn- und Mobilitätsverhalten haben.

\section{- Wissen ist Macht}

Wer einen anderen Konsum praktizieren will, braucht „objektive“ Informationen über Produkte, Herstellungsweise und Unternehmenspolitik. So reagierten die Unternehmen auf das steigende Umweltbewußtsein in Deutschland mit einer Inflation umweltbezogener Werbung: Kaum eine Anzeige ohne „Bio“-Hinweis und kaum ein Werbefilm ohne „Öko“-Aspekt. Doch ob „schadstoffarm“ oder „umweltfreundlich“ die Flut dieser schlagwortgeprägten Werbung erzeugt eher das Gegenteil. Auf die Frage nach dem Informationswert verschiedener Broschüren zu sozialen und ökologischen Aspekten der Unternehmenspolitik schlagen die Veröffentlichungen von Umwelt- und Verbraucherorganisationen nach einer Emnid-Studie von 1993 die Unternehmenspublikationen um Längen. Schon heute sorgen Umwelt- und Verbraucherorganisationen für reichliche Informationsangebote. Aufwendig aufbereitete Informationen wie das Buch „Der Unternehmenstester“, der die Lebensmittelbranche beschreibt und bewertet, gehören ebenso dazu wie ungezählte Informationsbroschüren zu den unterschiedlichsten Themen von A wie Amalgam bis $\mathrm{Z}$ wie Zusatzstoffe. Im Einzelfall werden Verbände auch schon einmal selbst aktiv: So gehören u.a. der Tierschutzbund, BUND und VERBRAUCHER INITIATIVE zu den Trägern des NEULAND-Fleischprogramms, das Fleisch nach tiergerechten und umweltschonenden Bedingungen erzeugt.

\section{Wie weiter?}

Auf dem Kongress „Konsumwende - mehr Wohlstand für alle?" wies Frau Hansen (Professorin an der Universität Hannover) auf die Widersprüchlichkeiten im Marketing- und Konsumverhalten hin. So existieren einerseits Orientierungs- und Sinnverluste, andererseits gibt es neue Versuche der Sinnstiftung. Einerseits werden Glaubwürdigkeitsverluste durch Hinnahme von Widersprüchen oder Pendeln zwischen Optionen festgestellt, andererseits entwickeln neue Institutionen, wie z.B. Greenpeace, Glaubwürdigkeit.

Nachhaltiger Konsum braucht motivierte Verbraucher, die ihre Nachfragemacht einsetzen und dadurch Unternehmen zu einem veränderten Angebot zwingen bzw. ein geändertes Produktangebot durch ihren Kauf unterstïtzen. Von Seiten der Unternehmen werden Verbraucher und Organisationen verstärkt als Co-Produzenten einbezogen werden, um so ein zukunftsfähiges Angebot zu entwickeln und um Fehlentwicklungen zu minimieren. Dabei werden die Verbraucher mit erhöhten Preisen konfrontiert, da die ökologischen Kosten in die Preisgestaltung einfließen müssen.

Zusätzlich besteht die Gefahr einer Polarisierung der Gesellschaft zwischen denjenigen, die auf höchstem Niveau Verzicht und einen anderen Konsum predigen, und denjenigen mit unerfüllten Konsumwünschen, aber nur begrenzten Möglichkeiten, diese Wünsche realisieren zu können. An dieser Stelle ist der Konflikt vorprogrammiert, und hier wird sich erweisen, ob bzw. wie ökologischer und sozialer Konsum funktionieren wird.

\section{Der Autor}

Georg Abel ist Geschäftsführer der VERBRAUCHER INITIATIVE, dem Bundesverband kritischer Verbraucherinnen und Verbraucher

Kontakt: Breitestr. 51, 53111 Bonn,

Tel. (0228) 726-3314, Fax -3399 
(c) 20I0 Authors; licensee IÖW and oekom verlag. This is an article distributed under the terms of the Creative Commons Attribution Non-Commercial No Derivates License (http://creativecommons.org/licenses/by-nc-nd/3.o/), which permits unrestricted use, distribution, and reproduction in any medium, provided the original work is properly cited. 